\title{
Postural Modifications and Neuronal Excitability Changes Induced by a Short-Term Serotonin Depletion during Neonatal Development in the Rat
}

\author{
Jean-François Pflieger, François Clarac, and Laurent Vinay \\ Développement et Pathologie du Mouvement, Centre National de la Recherche Scientifique, F-13402 Marseille, Cedex \\ 20, France
}

Serotonin (5-HT) plays an important role both in the development and in the recovery of locomotion after spinalization in vertebrates. We investigated the contribution of the serotonergic system to the maturation of the lumbar motoneurons and networks in the neonatal rat. A 5-HT synthesis inhibitor, p-chlorophenylalanine (PCPA), was administered daily from the first postnatal day (P0) onward. This protocol depleted serotonin in the spinal cord within 3-4 d, as demonstrated by immunohistochemistry. PCPA-treated rats exhibited postural changes characterized by lesser flexion at the knee and ankle levels and lesser extension of the hip. Posture was asymmetric, suggesting possible deficits in the interlimb coordination. Intracellular recordings were made at P3-5 from motoneurons innervating different hindlimb muscles, using the in vitro brainstem-spinal cord-nerve-attached preparation. In PCPA-treated rats, the conduction velocity of motoneurons was increased, and their excitability was decreased (because of higher rehobase and input conductance) compared with sham animals. In accordance with postural observations, changes were more pronounced in hip extensor/knee flexor than in ankle extensor motoneurons. The maturation of repetitive firing properties was stopped by PCPA treatment, although PCPA, applied in vitro, had no effect on membrane properties. The spontaneous endogenously generated activity, which is a characteristic of immature networks, was increased in PCPA-treated rats, suggesting that developing lumbar networks are sensitive to 5-HT levels. Serotonin may play a critical role during development in regulating the balance between the excitability of motoneurons and that of interneurons. Interneuronal excitability is crucial for the activity-dependent development of spinal cord networks.

Key words: development; motoneurons; motor behaviors; posture; rat; serotonin; spinal cord; spontaneous activity
It is well established that serotonin (5-HT) plays important roles in motor control in adult vertebrates (Jacobs and Fornal, 1993; Kiehn et al., 1996; Jankowska et al., 2000; Schmidt and Jordan, 2000). During development in the rat, serotonergic fibers arrive in the lumbar spinal cord around embryonic day (E) 16 and start to innervate the gray matter by E17 (Bregman, 1987; Rajaofetra et al., 1989). An adult-like pattern of projections is observed on postnatal day (P) 21. Because of their early arrival in the lumbar enlargement, these afferents are in a position to regulate several developmental processes. Numerous studies showed that 5-HT has ubiquitous tropic and trophic effects on the early development of neurons and synapses (Lauder, 1993; Okado et al., 1993; Mazer et al., 1997; Buznikov et al., 2001).

The role of 5-HT in the development of spinal neuronal networks has been scarcely studied. The ingrowth of serotonergic axons to the spinal cord plays a critical role in locomotor burst development in the tadpole (Sillar et al., 1995). Increased 5-HT levels during ontogeny in transgenic monoamine oxidase A-deficient mice alters the stability of respiratory (Bou-Flores et al., 2000; Burnet et al., 2001) and locomotor (Cazalets et al., 2000) rhythms. 5-HT depletion leads to impaired interlimb coor-

Received Jan. 2, 2002; revised April 4, 2002; accepted April 8, 2002.

J.-F.P. was supported by the Fondation pour la Recherche Médicale (France), the Fonds pour la Recherche en Santé du Québec, and the Natural Sciences and Engineering Research Council of Canada.

Correspondence should be addressed to Dr. L. Vinay, Développement et Pathologie du Mouvement, Centre National de la Recherche Scientifique, 31 chemin Joseph Aiguier, F-13402 Marseille, Cedex 20, France. E-mail: vinay@dpm.cnrs-mrs.fr. Copyright (C) 2002 Society for Neuroscience $0270-6474 / 02 / 225108-10 \$ 15.00 / 0$ dination during locomotion in the neonatal rat (Myoga et al., 1995; Nakajima et al., 1998).

The first postnatal week is a key period for the motor development of the rat. The animal switches gradually from a prone posture at birth to a quadruped stance with sustained extension of the ankle (Bolles and Woods, 1964; Geisler et al., 1993; Brocard et al., 1999a). At least two mechanisms may account for the development of posture during this period: the arrival of descending pathways (Kudo et al., 1993; Lakke, 1997; Brocard et al., $1999 \mathrm{~b})$ in the lumbar enlargement and the maturation of motoneurons (Fulton and Walton, 1986). Maturation does not proceed simultaneously in all motor pools and muscles. For instance, ankle flexor motoneurons acquire repetitive firing properties earlier than ankle extensor motoneurons do (Vinay et al., 2000a). Spontaneous activity can be recorded in vitro from the lumbar segments of the spinal cord isolated from fetal or neonatal rats (Nishimaru et al., 1996; Nakayama et al., 1999; Fellippa-Marques et al., 2000). This endogenously generated activity is believed to play an important role in the maturation of networks (for review, see Vinay et al., 2000b). The aim of the present study was to investigate the contribution of serotonin to the maturation of lumbar motoneurons and networks. We blocked 5-HT synthesis from birth onward (Myoga et al., 1995; Nakajima et al., 1998) and investigated the effects of 5-HT depletion several days later by means of behavioral, electrophysiological, and immunohistochemical techniques. The in vitro spinal cord preparation, with motor nerves of the hindlimb attached, enabled us to identify the recorded motoneurons functionally and thereby to correlate their properties with the observations made in vivo. 


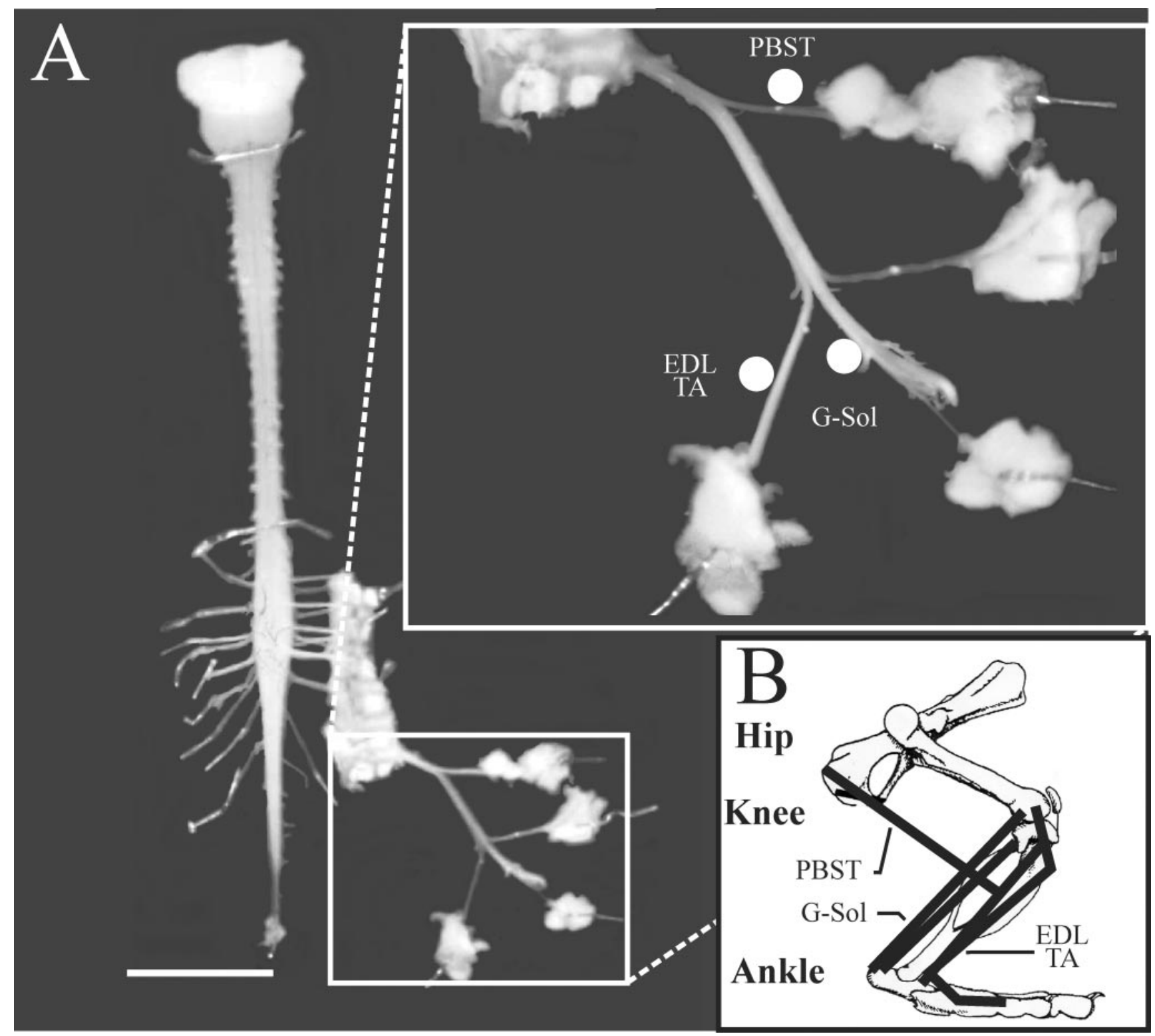

Figure 1. In vitro brainstem-spinal cord-sciatic nerve preparation. $A$, Ventral view of the preparation used in this study. The inset shows a higher magnification of the different nerve branches left attached to the spinal cord that are stimulated to identify motoneurons functionally. Filled circles indicate the approximate location of stimulating electrodes. Branches were identified by their muscular target $(B)$. $B$, Schematic lateral view of the rat hindlimb showing the location and insertions of different muscles. EDL, Extensor digitorum longus; $G-S o l$, gastrocnemius-soleus; PBST, posterior biceps, semitendinosus; $T A$, tibialis anterior. Scale bar, $1 \mathrm{~cm}$.

Some results have been published previously in abstract form (Pflieger et al., 2000).

\section{MATERIALS AND METHODS}

A total of 97 Wistar rats aged from P0 (defined as the first $24 \mathrm{hr}$ after birth) to P11 were used. Each litter was divided into two groups of approximately the same number of pups: an experimental group, injected intraperitoneally with $p$-chlorophenylalanine methyl ester (PCPA) [Sigma, St. Louis, MO; $300-400 \mathrm{mg} / \mathrm{kg}$ diluted in phosphate buffer (PB); protocol adapted from Myoga et al. (1995); Nakajima et al. (1998)], and a sham group, injected daily with PB. All surgical and experimental procedures were made to minimize animal suffering and conformed to the guidelines from the French Ministry for Agriculture and Fisheries, Division of Animal Rights.

Behavioral observations. Injected specimens were weighed and observed daily, and qualitative observations were made. Behavioral deficits induced by PCPA were evaluated at P4 -5 on sham $(n=9)$ and PCPAtreated $(n=9)$ specimens from two litters. The median toe and the heel of both hindfeet were labeled with a dark pen. Each animal was then placed on a horizontal glass, previously warmed by means of an infrared lamp. After the animal rested for $1 \mathrm{~min}$, its posture was recorded from below with a video camera (Sony, CCD-TR3100E; 25 frames per second). Recordings never lasted more than 2 min. Experiments were performed between 1:00 and 3:00 P.M. and at least $18 \mathrm{hr}$ after the last injection to avoid possible acute effects of PCPA. The first minute of recording, in the absence of any locomotor movement, was considered for analysis. The position of the markers relative to body axis, defined by the line from the navel to the anus, was sampled every $10 \mathrm{sec}$.

Electrophysiology. These experiments were made at P3-5, 14-20 hr after the last injection (shams, $n=11$; PCPA-treated pups, $n=24$ ). Animals were anesthetized by hypothermia. They were then decerebrated at a post-collicular level, eviscerated, and pinned down onto a Petri dish. Dorsal craniotomy and laminectomy were performed, and the brainstem and spinal cord were removed. The sciatic nerve and some of its branches were carefully dissected and left attached on one side of the cord (Fig. 1A). These branches innervated the ankle flexor muscles 
[extensor digitorum longus (EDL) and tibialis anterior (TA) and their synergist digit flexors], the ankle extensor muscles [gastrocnemius (G) and soleus (Sol), and their synergist digit extensors], and the knee flexor/hip extensor muscular group [posterior biceps and semitendinosus (PBST)] (see Fig. 1B). The preparation was then pinned down with the ventral side up in the recording chamber. The pia was removed on one side of the lumbar enlargement (from L3 to L6) to enable the penetration of microelectrodes. All dissection and recording procedures were performed under continuous perfusion with saline solution containing (in $\mathrm{mm}$ ): $\mathrm{NaCl} 130, \mathrm{KCl} 4, \mathrm{CaCl}_{2} 3.75, \mathrm{MgSO}_{4} 1.3, \mathrm{NaH}_{2} \mathrm{PO}_{4} 0.58, \mathrm{NaHCO}_{3}$ 25 , glucose 10 ; oxygenated with $95 \% \mathrm{O}_{2} / 5 \% \mathrm{CO}_{2} ; \mathrm{pH}$ adjusted to 7.4 and temperature to $24-27^{\circ} \mathrm{C}$.

Monopolar stainless steel electrodes were placed in contact with the nerve branches or roots and insulated with Vaseline for stimulation. The distance between the spinal cord and the electrodes was measured before insulation for subsequent calculation of conduction velocity. Intracellular potentials were recorded from lumbar motoneurons by means of glass microelectrodes ( $2 \mathrm{M} \mathrm{K}$-Acetate; $75-120 \mathrm{M} \Omega$ ) in the bridge or discontinuous current-clamp (DCC) modes (Axoclamp 2B amplifier; Axon Instruments, Union City, CA). During DCC recordings, the headstage output was monitored continuously to properly adjust the capacitance compensation and sampling rate $(1.5-3 \mathrm{kHz})$. Motoneurons were identified functionally by their antidromic response to stimulation of either of the different branches of the sciatic nerve and thereby divided into three groups (EDL-TA, G-Sol, and PBST). Only neurons exhibiting a stable resting potential of at least $-60 \mathrm{mV}$ for $>20$ min were considered for analysis. Rheobase was defined as the minimum current intensity necessary to fire the cell. The input resistance was measured by injecting moderate current pulses $(-0.5$ to $+0.5 \mathrm{nA}, 500 \mathrm{msec})$, which did not produce any rectification. Conductance was calculated as the reciprocal of input resistance. Repetitive firing properties were studied by injecting depolarizing square-pulses (500 msec duration) of different amplitudes, normalized to rheobase. All data were collected through a Digidata 1200 interface connected to a PC computer, and pClamp 8.0 software was used for digital acquisition $(5-20 \mathrm{kHz})$ and data analysis (both interface and software from Axon Instruments).

Immunohistochemistry. Immunochemical experiments were conducted to confirm that PCPA depletes 5-HT in the lumbar cord. Thirteen rats (P3-4) were prepared for in vitro studies as described above, although only the caudal spinal cord and roots were kept (L1-S2). In eight cases (noninjected controls, $n=2$; shams, $n=3$; PCPA-treated rats, $n=3$ ), the cord was directly immersed in $4 \%$ paraformaldehyde (PFA) after dissection. For the remaining cases (shams, $n=2$; PCPA-treated rats, $n=3$ ), the cord was gently dried, and crystals of Fluorescein-conjugated dextran amines (FDA) (3000 molecular weight; Molecular Probes) were applied to the L5 ventral root on one side. After 5-10 min, preparations were then reimmersed in circulating saline solution for $13-15 \mathrm{hr}$ of retrograde transport before fixation in PFA. One P0 animal was used for intracellular labeling of functionally identified motoneurons. Two neurons were impaled with a micropipette filled with Lucifer yellow (Sigma; $5 \%$ in $1 \mathrm{M} \mathrm{LiCl}$ ), identified by their antidromic response to stimulation of the ankle flexor and extensor branches, respectively. Negative squarepulses were delivered $(1.5-2.5 \mathrm{nA} ; 500 \mathrm{msec}$; $1 \mathrm{~Hz})$ for $30-40 \mathrm{~min}$ to fill the cells with the dye. The spinal cord was then fixed as described above.

After fixation, specimens were embedded in $4 \%$ agarose (low gelling temperature, diluted in distilled water; Sigma) and cut, transversally or horizontally, at $50 \mu \mathrm{m}$ on a vibratome. Sections were mounted on slides. After preincubation with normal goat serum (3\% in PB; Sigma), they were incubated overnight with rabbit 5-HT antiserum (dilution 1:5000; Sigma) followed by goat anti-rabbit IgG coupled to tetramethyl rhodamine isothiocyanate (TRITC) (dilution 1:100; Jackson ImmunoResearch, West Grove, PA). To serve as controls, some sections from noninjected and sham specimens were processed as above except that the rabbit antiserum was not added to the incubation bath. After rinse, sections were dehydrated in increasing concentrations of ethanol (50$100 \%)$ and cleared with methyl salicylate (Merck, Darmsadt, Germany). Slides were coverslipped using Fluoromount (BDH, Poole, UK) as mounting medium.

Sections were observed on a Zeiss microscope equipped for fluorescence (Axiophot) and microphotographed (FujiFilm RSP 1200 ASA). When needed, pictures were digitized with a scan (Epson Perfection 124OU). Corel Photo-Paint 8.0 software was used to adjust contrast and convert color microphotographs to grayscale.

Statistical analysis. Results are given in the text and figures in the form of mean \pm SEM. Student's $t$ test was used for statistical analysis when two groups were compared (Graphpad Prism 2 Software).

\section{RESULTS}

\section{PCPA depletes serotonin in the lumbar spinal cord}

The development of 5-HT projections to the spinal cord has been described extensively in Sprague Dawley rats (Bregman, 1987; Rajaofetra et al., 1989; Nakajima et al., 1998; Tanaka et al., 1992), and the blockade of 5-HT synthesis in the rat central nervous system by PCPA injection is well known (Koe and Weissman, 1966; Tohyama et al., 1988; Myoga et al., 1995; Nakajima et al., 1998). We nevertheless checked the presence of 5-HT immunoreactivity, at birth, in the lumbar ventral horn in the Wistar strain and whether the protocol we used resulted in a rapid decrease of 5-HT immunoreactivity in the spinal cord. At birth, serotonergic fibers are present in the vicinity of motoneuronal pools in the lumbar cord. Figure 2B1,2 shows an EDL-TA motoneuron filled with Lucifer yellow. Numerous 5-HT-immunoreactive fibers (arrows) were closely apposed to the neuron dendrites (Fig. 2B1) and soma (Fig. 2B2). The same environment rich in 5-HTimmunoreactive fibers was found for a G-Sol motoneuron (data not shown). At P3-4, labeled fibers were found in all lumbar segments in sham specimens, distributed in the ventral and lateral funiculi and throughout the gray matter as described previously (Bregman, 1987; Rajaofetra et al., 1989; Nakajima et al., 1998). These fibers possessed numerous varicosities that were observed close to the motoneurons retrogradely labeled with FDA (data not shown). In PCPA-treated animals, the number of 5HTimmunoreactive fibers and varicosities was markedly reduced. The remaining labeled fibers were thin and more often found medially in the ventral funiculus (data not shown). The gray matter was devoid of 5HT-immunoreactive fibers in all but two specimens. In the latter, the fibers were very sparse in the area of motor pools (Fig. 2C2). In Figure $2 C$, photomicrographs of similar regions of the L5 ventral horn in sham-injected (Fig. 2C1) and PCPA-injected (Fig. 2C2) specimens (both at P4) show that 5-HT-immunoreactive fibers (arrows) were observed close to motoneuron cell bodies (arrowheads) only in the sham animal. Three to four daily injections of PCPA therefore deplete 5-HT markedly.

\section{PCPA induces postural instability, reduces hip extension, and causes a hyperextension at the knee and ankle joints}

Sham- and PCPA-treated neonates could not be differentiated during the first three postnatal days ( $\mathrm{P} 0-2)$. From $\mathrm{P} 2$ onward, the daily weight increase of PCPA-treated animals was reduced compared with sham animals (Fig. 3) $(n=23$ shams and 23 PCPAtreated animals). The weight increase of sham (regression line slope: $1.45 \pm 0.07)$ and PCPA-treated (slope: $0.86 \pm 0.07)$ rats differed significantly ( $p<0.001$ ) between P0 and P6. By contrast, the weight increase was similar between P6 and P9 as soon as the PCPA treatment stopped $(p=0.97)$. All of the experiments described below were performed at P3-5, when weight increase curves started to diverge. Similar reduced body weights and weight increases have been described after prenatal or postnatal injections of PCPA in Sprague Dawley rats (Myoga et al., 1995; Nakajima et al., 1998).

At around P3, a remarkable behavior exhibited by PCPAtreated was a hyperextension of the hindlimbs at the ankle and knee levels associated with a hip flexion (Fig. 4A). This movement lasted 1-5 sec and concerned a single limb in younger 

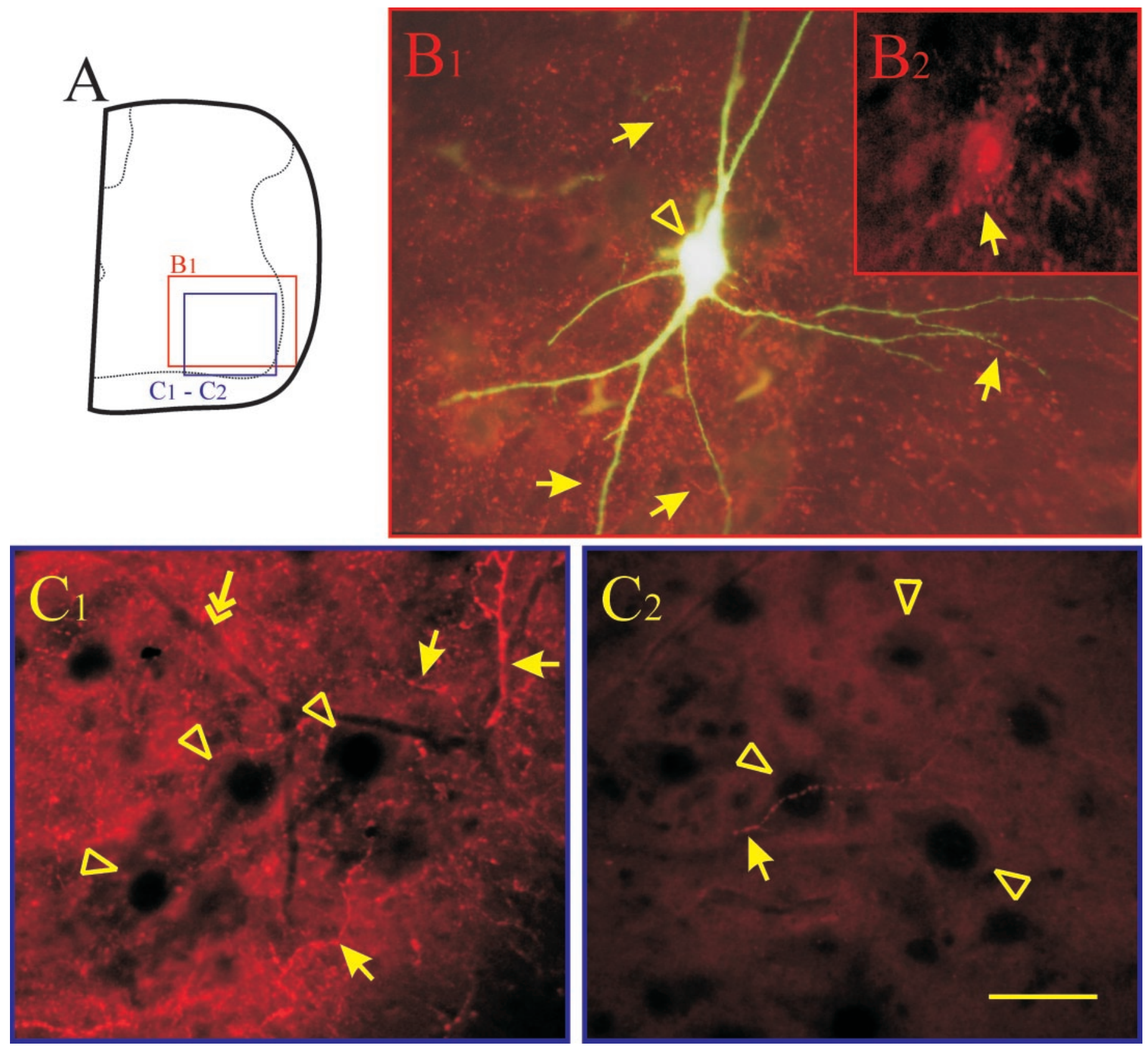

Figure 2. Immunohistochemical confirmation that PCPA injections deplete serotonin in the spinal cord of neonatal rat. $A$, Schematic transverse section through the right hemicord of a lumbar segment, indicating approximately the location of the microphotographs in $B$ and $C$ (note that these are from different specimens). Dorsal is $u p$, and lateral is to the right. $B$, Serotonergic fibers found close to lumbar motoneurons in a P0 rat. B1, Transverse section of the lumbar (L5) spinal cord, showing a Lucifer yellow-labeled motoneuron (arrowhead), previously identified as innervating the ankle flexor muscle, and TRITC-labeled (red) serotonergic varicosities and fibers (arrows). B2, Single fluorescent filter exposition of the same neuron showing a serotonergic fiber (arrow) in close proximity to the cell soma. Note that only the fluorescent filter for TRITC was used but that the outlines of the cell soma and nuclei are visible because of their intense labeling. $C$, Depletion of serotonin induced by PCPA injections. Transverse sections at lumbar (L5) level in P4 sham-injected (C1) and PCPA-injected (C2) animals. Serotonergic fibers (arrows) close to large cell bodies, presumably motoneurons (unlabeled; arrowheads), are numerous in the sham but were very sparse in the PCPA-treated specimen. The double-headed arrow in C1 indicates a blood vessel. Scale bar, $50 \mu \mathrm{m}$.

animals, whereas it was sometimes observed bilaterally in older and more affected specimens. In a given animal, the unilateral extension was observed on the left or the right side, indiscriminately. The posture displayed spontaneously at P4-5 by PCPAinjected animals and shams, after they were placed on a surface (Fig. 4A), was investigated by comparing the position of the markers at the toe and heel levels relative to body axis (Fig. $4 B$ shows six successive positions of the markers, labeled 1-6, sampled every $10 \mathrm{sec}$ ). The angle between the foot axis (toe-heel) and the body axis (navel-anus line) was measured for the left and right feet (Fig. $4 B, \alpha l$ and $\alpha r$, respectively). These angles were used to estimate the "postural symmetry" $(\alpha \mathrm{l}-\alpha \mathrm{r}$, in absolute value; a perfectly symmetrical placement of the feet would give a zero angle) and the "postural stability" $(\alpha \mathrm{l}+\alpha \mathrm{r}$; the larger this 


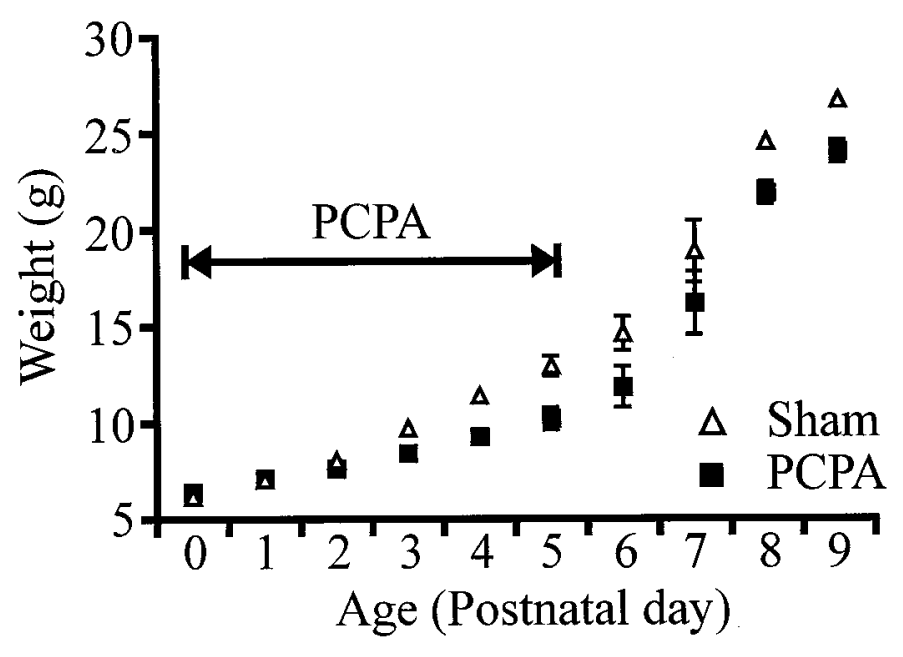

Figure 3. Age-related weight increase of sham and PCPA-treated rats. From P3 onward, PCPA-treated animals had a lower daily weight increase than shams ( $n=23$ for both groups). The weight increase returned to normal soon after the last PCPA injection (P5); the horizontal bar indicates the duration of the treatment.

summation, the larger the basis of support and the better the control of balance). We found that the "postural asymmetry" was larger $(p<0.05)$ (Fig. 4C1) and the postural stability was smaller $(p<0.05)$ (Fig. 4C2) in PCPA-treated animals than in shams. The unstable asymmetric posture associated with phasic unilateral extensions of the hindlimbs sometimes led PCPA-treated animals to turn themselves upside-down. A third notable difference between the two animal groups concerned the rostrocaudal placement of the feet, estimated by measuring the projection, at right angles, of the toes onto the body axis. This projection was significantly more rostral $(p<0.01)$ (Fig. 4C3) in PCPA-treated specimens than in shams, being ahead and behind the navel, respectively. Both the spontaneous movements and the posture at rest suggest a deficit in hip extension after 5-HT depletion. Altogether, these observations show that posture was less efficient to counteract gravity and stabilize the body in PCPA-treated animals than in shams.

\section{The excitability of motoneurons is decreased after serotonin depletion}

In vitro experiments were performed on the isolated spinal cord preparation to investigate the possible physiological changes that may account for the postural deficits observed in PCPA-treated animals at P3-5. In 35 animals (shams, $n=11$; PCPA-treated pups, $n=24)$, intracellular recordings were made from motoneurons innervating the ankle flexor muscles (EDL-TA), the ankle extensor muscles (G-Sol), and the mixed knee flexor/hip extensor muscular group (PBST) (Fig. 1).

\section{Motoneuron properties}

When the passive membrane properties of all recorded motoneurons from the two groups ( $n=22$ in shams; $n=49$ in PCPAtreated animals) were compared, no difference was observed in the resting potential (Table 1). Significant increases in both input conductance $(p<0.01)$ (Fig. $5 A)$ and rheobase $(p<0.05)$ (Fig. $5 B$ ) were observed in PCPA-treated rats, indicating an overall decreased excitability of motoneurons after 5-HT depletion. No correlation was found between rheobase and input conductance in motoneurons of the sham group $(r=0.20 ; p>0.05)$ (Fig. $5 C$ ). By contrast, a significant and positive correlation between these parameters was observed in the PCPA-treated group $(r=0.78$; $p<0.001$ ) (Fig. 5C). This reflects a shift in the motoneuronal population from low rheobase and conductance toward higher rheobase and input conductance in 5-HT-depleted specimens. A similar shift was observed previously in rat motoneurons between P0-2 and P6-8 (Seebach and Mendell, 1996).

We compared excitability changes in the three populations of motoneurons. The increase in input conductance was significant only for PBST motoneurons $(p<0.01)$ (Fig. 5D). G-Sol motoneurons were the least affected population. A nonsignificant trend toward a higher rheobase was observed for all motoneurons in PCPA-treated animals compared with those in shams. The most and the least pronounced differences were found for EDL-TA and G-Sol motoneurons, respectively (Table 2). These results suggest that the depletion of 5-HT caused a decrease in excitability more important in PBST and EDL-TA than in G-Sol motoneurons.

Conduction velocities of motoneurons were also affected in PCPA-treated rats (Fig. 6A). A higher conduction velocity ( $p<$ 0.05 ) was observed for EDL-TA and PBST populations, whereas no significant difference was observed for G-Sol motoneurons. When all motoneurons are pooled, a significant and positive correlation is found between conduction velocity and input conductance in the two groups (Fig. 6B, solid line, PCPA-treated; dashed line, sham). The stronger correlation in PCPA-treated may be related to the wider range of input conductance values in these specimens.

Depletion of 5-HT affected the capability of motoneurons to fire repetitively. In the neonatal rat, the response of neurons to the injection of $500 \mathrm{msec}$ depolarizing pulses can be divided into three patterns (Vinay et al., 2000a): no repetitive firing (single or double action potential at the pulse onset; Fig. 7A, top trace), transient discharge generated during the first half of the pulse (Fig. 7A, middle trace), and sustained discharge throughout the depolarization (Fig. 7A, bottom trace). These patterns have been proposed to represent three successive stages in the development of firing (for review, see Vinay et al., 2000b). They are not influenced by the intensity of the depolarizing current, i.e., a given neuron does not switch from a pattern to another with increasing current. A previous study showed that, at birth, only $50 \%$ of the G-Sol motoneurons exhibit repetitive firing, $21 \%$ exhibit transient firing, and $29 \%$ are incapable of repetitive firing (Fig. 7B) (Vinay et al., 2000a). The fraction of motoneurons exhibiting sustained firing increases with age. At P3-5, this fraction was higher in sham (82\%) than in PCPA-treated rats $(61 \%)$ (Fig. 7B). "Immature" neurons, incapable of repetitive firing, were few in shams $(9 \%)$, whereas they were still represented primarily in PCPA-treated rats $(26 \%)$ (Fig. $7 B)$. We compared the firing frequency of those neurons exhibiting sustained firing by injecting depolarizing current pulses of varying strengths. No difference was observed in the mean number of action potentials (Fig. 7C). These results suggest that 5-HT depletion blocked the acquisition of repetitive discharge properties in these motoneurons but did not affect the firing rate once this capability had been acquired.

All intracellular recordings were made 14-20 hr after the last injection of PCPA. We nevertheless tested whether PCPA was able to affect motoneuronal properties. Membrane properties of four motoneurons in three preparations were compared before and at least $15 \mathrm{~min}$ after bath application of PCPA (400 $\mu \mathrm{M}$ corresponding to $\sim 100 \mathrm{mg} / \mathrm{kg}$ ). No change was observed in either the rheobase or the input resistance (Fig. $8 A$ ) or the repetitive 

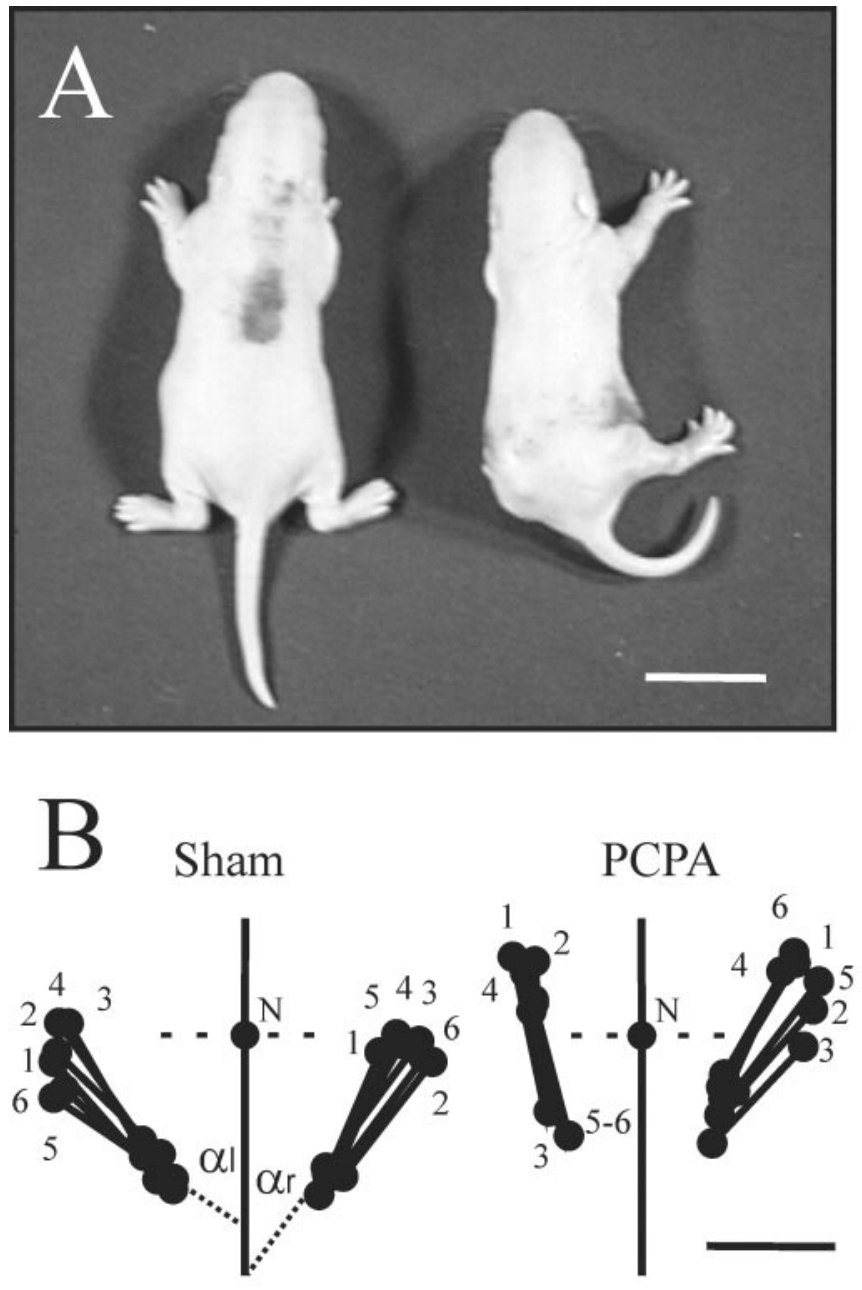

$\mathrm{C}_{1}$

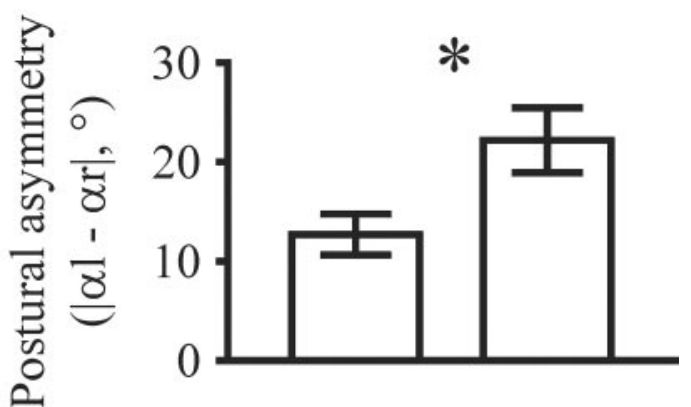

$\mathrm{C}_{2}$
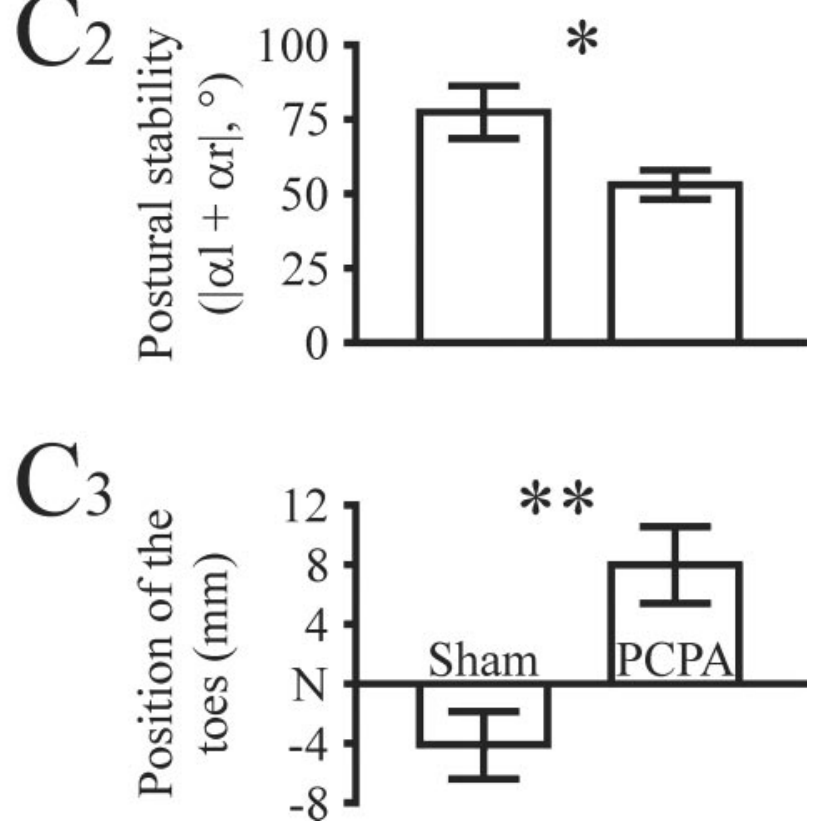

Figure 4. Postural deficits in PCPA-treated rats. A, Dorsal view of a sham-injected (left) and a PCPA-injected (right) animal (P6), illustrating the difference in posture between the two groups. The postural asymmetry of the PCPA-treated specimen is demonstrated by a hyperextension of the right hindlimb. $B$, Graphs illustrating the positions of hindfeet relative to the navel ( $N$, horizontal dotted line) and the body axis (vertical line). Each graph represents six observations (labeled from 1 to 6$)$. Angles between the body axis and the left $(\alpha l)$ or the right ( $\alpha r$ ) foot axis were measured. $C$, Postural asymmetry $(C 1)$, postural stability $(C 2)$, and rostrocaudal position of the toes relative to the navel (C3) (positive values mean rostral) for the sham-injected (left columns; $n=9$ ) and PCPA-injected (right columns; $n=9$ ) specimens. ${ }^{*} p<0.05$; ${ }^{* *} p<0.001$; Student's $t$ tests. Scale bars, 2 cm.

\begin{tabular}{|c|c|c|c|c|}
\hline & Whole population & EDL-TA & G-Sol & PBST \\
\hline Sham & $-73.2 \pm 4.2,29$ & $-71.7 \pm 2.2,7$ & $-74.5 \pm 0.8,12$ & $-72.8 \pm 1.3,10$ \\
\hline PCPA-treated & $-73.4 \pm 0.5,61$ & $-73.4 \pm 1.3,18$ & $-73.2 \pm 0.9,25$ & $-72.5 \pm 1.1,19$ \\
\hline
\end{tabular}

Values are expressed as means \pm SEM, number of neurons.

firing (Fig. $8 A, B$ ). These results suggest that PCPA per se does not modify the excitability or firing properties and that the changes described previously were likely caused by the 5-HT depletion.

\section{The endogenously generated spontaneous activity is increased after serotonin depletion}

Spontaneous activity is an intrinsic characteristic of developing networks (for review, see O'Donovan, 1999; Ben-Ari, 2001). It is believed to participate in the development of neurons and networks. Figure $9 A$ illustrates the spontaneous activity in a PBST motoneuron in a 5-HT-depleted specimen
(P4). The postsynaptic potentials observed at the resting potential (Fig. 9A1) are mixed, made of both excitation and inhibition, as demonstrated by steadily depolarizing the neuron (Fig. 9A2). Figure $9 B$ shows the area under bursts of postsynaptic potentials, above the resting potential, in all the recorded motoneurons in which spontaneous activity was investigated. The activity was increased in PCPA-treated rats compared with shams (shams, $278.9 \pm 35.88 \mathrm{mV} / \mathrm{sec}, n=9$; PCPA-treated rats, $690.9 \pm 105.5 \mathrm{mV} / \mathrm{sec}, n=9 ; p<0.01$ ). Postsynaptic potentials $>5 \mathrm{mV}$ were more numerous after 5 -HT depletion than in shams $(13.3 \pm 2.3$ potentials per 

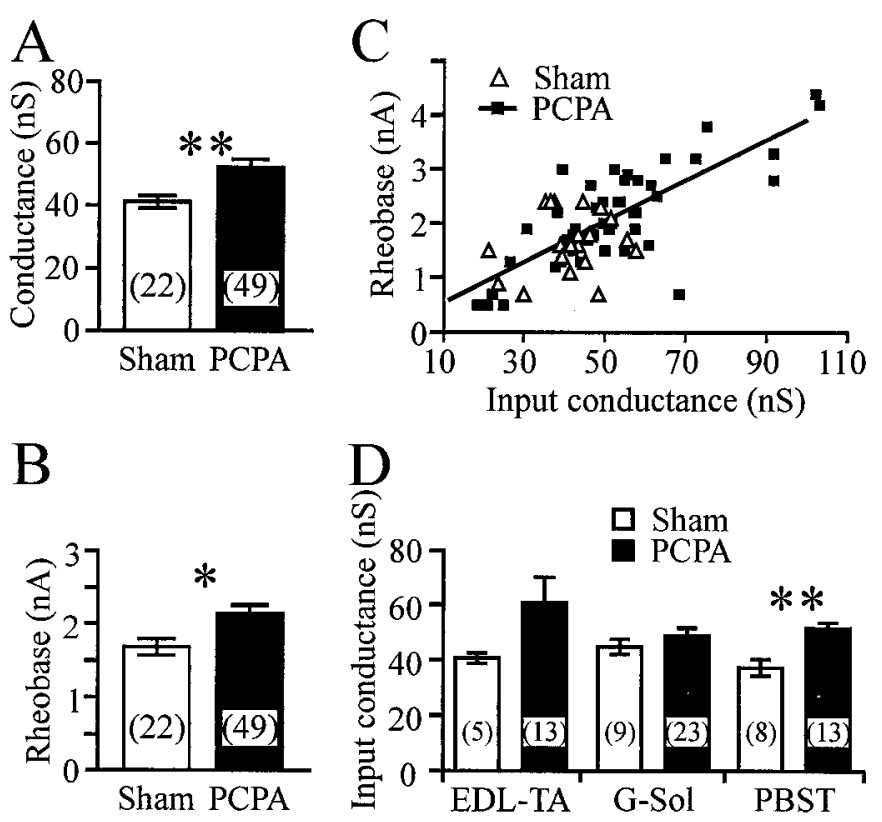

Figure 5. Changes in motoneuron excitability in PCPA-treated rats. Motoneuron conductance $(A)$ and rheobase $(B)$ are higher in PCPAtreated rats than in shams. $C$, Motoneuron rheobase and input conductance are positively correlated only in PCPA-treated specimens. $D, A v-$ erage conductance in functionally identified motoneurons of sham- or PCPA-treated specimens. Graphs show a trend toward higher rheobase and input conductance after PCPA treatment. The motoneurons innervating the ankle extensor muscles are the least affected. The number of neurons is indicated in brackets. ${ }^{*} p<0.05 ;{ }^{* *} p<0.01$; Student's $t$ tests.

Table 2. Rheobase (nA) of motoneurons from sham or PCPA-treated specimens at $\mathrm{P3}-5$

\begin{tabular}{lllll} 
& $\begin{array}{l}\text { Whole } \\
\text { population }\end{array}$ & EDL-TA & G-Sol & PBST \\
\hline Sham & $1.7 \pm 0.5,22$ & $1.4 \pm 0.3,5$ & $1.7 \pm 0.1,9$ & $1.9 \pm 0.2,8$ \\
PCPA-treated & $2.1 \pm 0.1,49$ & $2.5 \pm 0.4,12$ & $1.8 \pm 0.1,23$ & $2.3 \pm 0.1,14$
\end{tabular}

Values are expressed as means \pm SEM, number of neurons.

minute, $n=9$, and $4.8 \pm 2.0, n=9$, respectively). The more intense activity in PCPA-treated specimens, despite the lower input resistance of motoneurons, suggests that the excitability of immature lumbar networks is increased after 5-HT depletion.

\section{DISCUSSION}

A depletion of 5-HT during a short developmental time window induced marked postural deficits characterized by a lesser flexion at the knee and ankle levels and lesser extension of the hip. Asymmetry of posture suggested deficits in the interlimb coordination [see also Myoga et al. (1995)]. Opposite effects were observed on the excitability of interneurons and motoneurons. Pools of motoneurons were not affected in the same way. A significant decrease in excitability was noted for the hip extensor/ knee flexor, whereas the ankle extensor motor pool was affected only slightly. Our results also suggest that 5-HT plays a critical role in the development of firing properties.

\section{Serotonin and the development of motoneurons}

The input conductance of motoneurons was higher in PCPAtreated rats than in sham animals, leading to an increased rheobase and a consequent reduction in cell excitability. Conductance and rheobase increase during the first postnatal weeks (Fulton and Walton, 1986; Seebach and Mendell, 1996). At least three mechanisms contribute to these age-related changes and may also account for the differences observed after 5-HT depletion: anatomic modifications, increased synaptic input, and maturation and distribution of ionic channels.

The morphological development of motoneurons has been described in the rat in two of the motor pools investigated in the present study: those innervating the soleus and those innervating the EDL-TA muscles (Westerga and Gramsbergen, 1992; Dekkers et al., 1994; Kerai et al., 1995). The most rapid growth of the soma occurs during the second postnatal week; changes in electrophysiological parameters from birth to P7 are not caused primarily by morphological development, which is limited during this period. However, theoretically, the reduced input resistance of motoneurons after 5-HT depletion might be caused by an increased growth of the soma or the dendritic tree, or both. Serotonin has been described as either inhibiting or promoting elongation of neurites (Haydon et al., 1984; Lauder, 1993; Sullivan et al., 2000). In the spinal cord, both the soma and dendrites of quadriceps femoris motoneurons are smaller at P0-5 after PCPA treatment during prenatal development compared with controls (Nakajima et al., 1996). In line with a promoting role for 5-HT on neurite outgrowth in the spinal cord, the dendritic tree of neonatal phrenic motoneurons is increased in monoamine oxidase A-deficient (Tg8) mice, which have a high 5-HT level during perinatal development, compared with the control strain (Bou-Flores et al., 2000). Okado et al. (1993) showed no effect of
Figure 6. Effects of serotonin depletion on the conduction velocity. $A$, Conduction velocities from functionally identified motoneurons from sham- and PCPA-treated animals at P3-5 (averages for the whole population are indicated by dashed and solid lines, respectively; no significant difference between the two populations; $p>0.05$ ). The number of neurons recorded in each group is indicated. ${ }^{*} p<0.05$; Student's $t$ test. $B$, Conduction velocity and input conductance are positively correlated in the two groups (PCPA-treated, solid line: $r=$ $0.72, p<0.001$; sham, dashed line: $r=0.51$, $p<0.05)$ but do not differ significantly from each other.
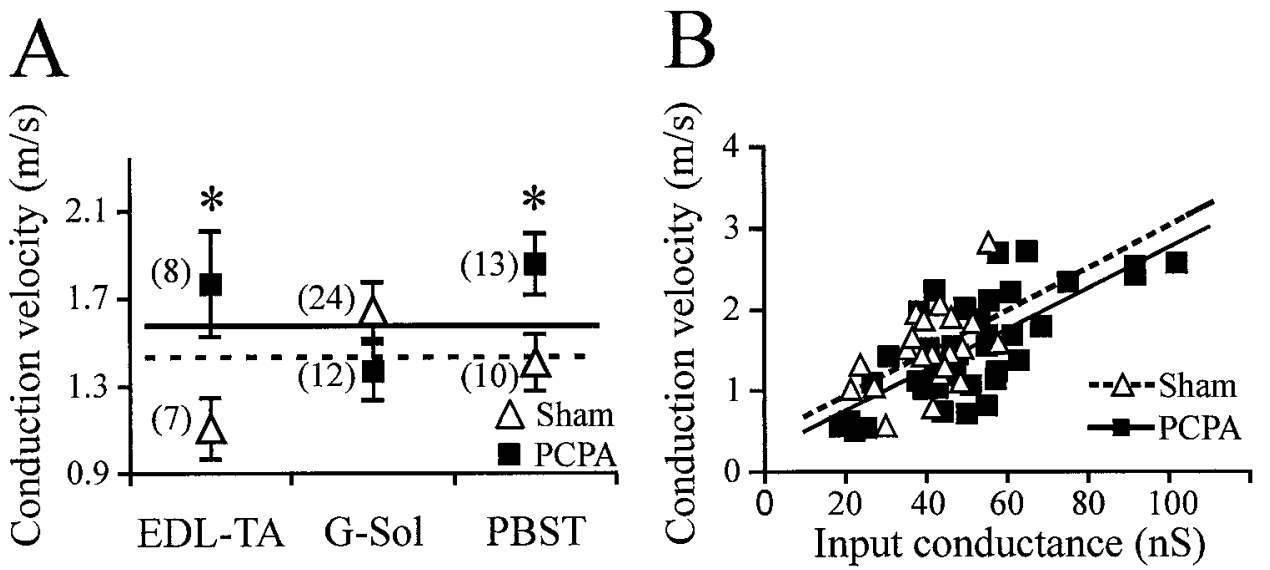
A

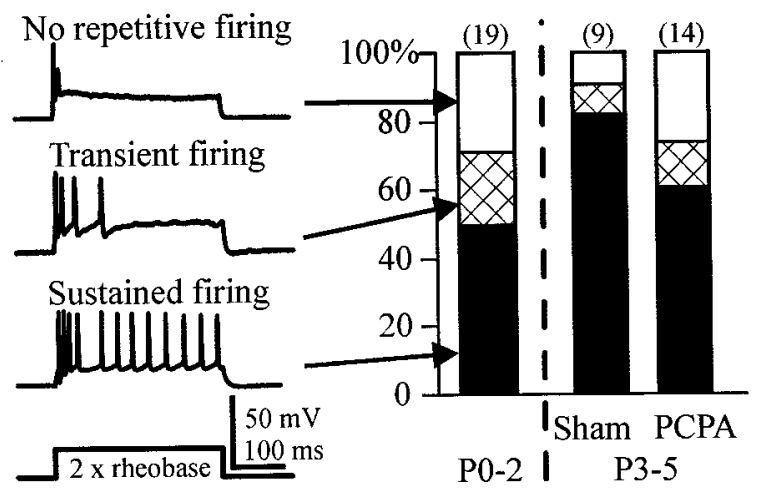

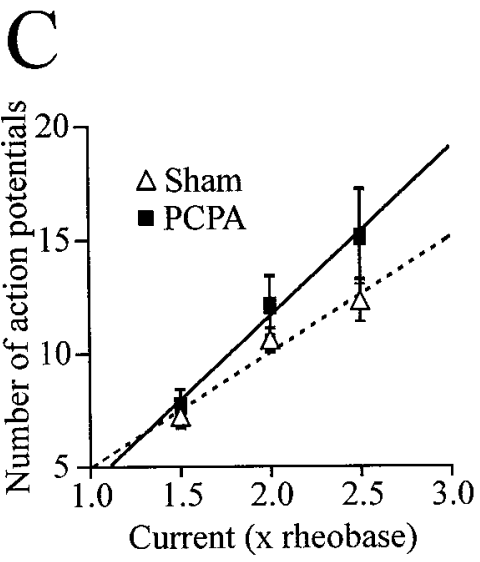

Figure 7. Serotonin depletion affects the maturation of repetitive firing properties. $A$, Discharge patterns observed in motoneurons after current injection at twice the rheobase: a single action potential or a doublet (top trace), a transient spike train (middle trace), or a sustained discharge throughout the pulse duration (bottom trace). Figure adapted from Vinay et al. (2000a). B, Proportion of G-Sol motoneurons generating the different patterns shown in $A$. The number of recorded neurons in each population is indicated at the top of each bar. The data for $\mathrm{P} 0-2$ were obtained from noninjected specimens (Vinay et al., 2000a). $C$, Mean number of action potentials (top graph) during 500 -msec-long repetitive discharge in G-Sol motoneurons of sham- and PCPA-

injected specimens, plotted against the magnitude of depolarizing current (normalized to rheobase). There is no statistical difference between the regression lines.

PCPA treatment on the pattern of dendritic structure during chick development. These data suggest that 5-HT depletion in our experiments had either no effect on the morphology of motoneurons or affected it (reduction of soma volume) in a way that cannot account for the reduction in input resistance.

Lumbar motoneurons are electrically coupled through gap junctions at birth, and this coupling decreases during the first postnatal week (Walton and Navarrete, 1991; Chang et al., 1999). Dye-coupling between pyramidal neurons is reduced after preincubation of acute cortical slices with 5-HT (Rörig and Sutor, 1996). Provided a similar modulatory effect on gap junctions occurs in the lumbar enlargement at the arrival of serotonergic pathways, a lower regression of the junctional coupling in PCPA-treated animals would make motoneurons less compact electrically (i.e., with a lower input resistance) than their counterparts in normal rats.

Increased spontaneous synaptic activity after PCPA treatment may cause a reduction in input resistance of motoneurons because synaptic transmission makes a significant contribution to the resting conductance of developing motoneurons [NunezAbades et al. (2000) on genioglossal motoneurons]. Most ionic currents in motoneurons increase, whereas others, such as the A-type $\mathrm{K}^{+}$current $\left(I_{\mathrm{A}}\right)$, decrease after birth (Berger et al., 1996; Gao and Ziskind-Conhaim, 1998). In addition, the distribution of those potassium channels underlying membrane resistance in the different somatodendritic compartments changes with age (Cameron et al., 2000). Whether the development and distribution of ionic currents are affected after 5-HT depletion is unknown. This is likely to be the case, however, as suggested by the arrest in the development of repetitive firing in PCPA-treated animals, although the mechanisms underlying the acquisition of these properties are not yet known (Vinay et al., 2000b). Our data confirm an earlier report that removing the influence of serotonergic projections prevents the acquisition of repetitive firing capability by spinal neurons (Sillar et al., 1995) in Xenopus tadpoles.

\section{Differential effects of serotonin depletion on motor pools}

Motoneurons innervating flexor muscles (EDL-TA and PBST) are located at the periphery of the ventral horn (laterally and ventrally, respectively), whereas G-Sol motoneurons are located deeper in the gray matter (Nicolopoulos-Stournaras and Iles, 1983). The ingrowth of 5-HT fibers in the gray matter of the rat lumbar cord starts at embryonic stages (E17-18) from the lateral and ventral funiculi and develops lateromedially and ventrodorsally (Rajaofetra et al., 1989; Tanaka et al., 1992). Consequently, at the time of birth, flexor motoneurons may be more densely innervated by serotonergic fibers than extensor motoneurons. Regional differences in the serotonergic innervation of spinal motor regions have been described in the rat (Steinbusch, 1981) and chicken (Kojima et al., 1988; Okado et al., 1988).

Serotonin depletion affected motoneurons differentially. Excitability changes were more pronounced in PBST and EDL-TA than in G-Sol motoneurons. By contrast, maturation of repetitive firing properties was stopped in the latter motor pool. These differential effects may depend on the level of development of motor pools at the time of the depletion, which may itself depend

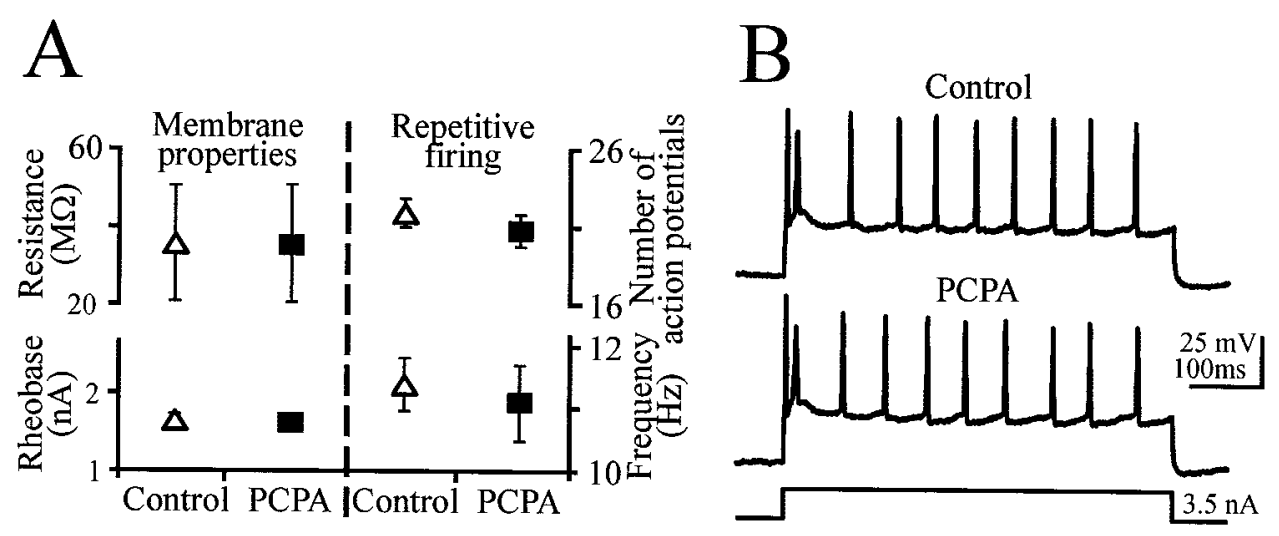

Figure 8. Bath application of PCPA did not change electrical properties of motoneurons. $A$, Comparison of some membrane properties (input resistance and rheobase, left column) and steady-state discharge properties (number of action potentials and discharge frequency, right column) before and during bath application of PCPA (400 $\mu \mathrm{M}, 100 \mathrm{mg} /$ $\mathrm{kg}$ ). Recordings were obtained from lumbar motoneurons $(n=5)$ at P3-5. B, Examples of discharge patterns before (top trace) and during (middle trace) PCPA application. Sustained firing was induced by depolarizing square pulses at twice the rheobase (bottom trace). 
Figure 9. Increase of spontaneous activity in serotonin-depleted animals. $A$, Spontaneous activity of a PBST motoneuron in a PCPA-treated specimen (P4). Intracellular recordings were made at resting potential (A1) and after a steady depolarization by current injection $(1.4 \mathrm{nA})$ to reveal the inhibitory and excitatory nature of the postsynaptic potentials (A2). B, Quantification of the spontaneous activity in motoneurons from sham-injected (triangle) or PCPAinjected (square) specimens. The area under postsynaptic potentials, above the resting potential, was measured over 30 consecutive $1 \mathrm{sec}$ bins and plotted against the time after dissection. A significant difference between mean spontaneous activity in PCPA-treated
B

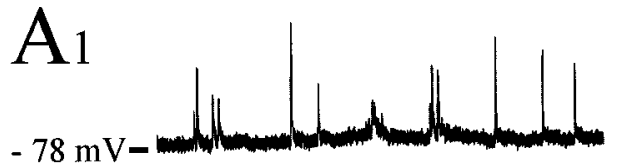

$\mathrm{A}_{2}$

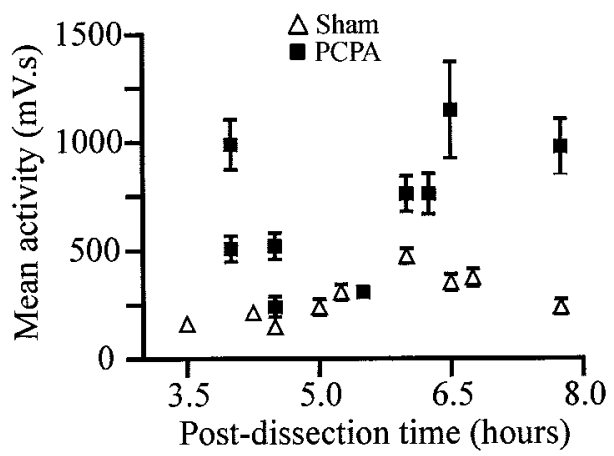
group.

on the intensity of the serotonergic innervation of the pools. The smaller excitability changes and delayed acquisition of repetitive firing property of $\mathrm{G}-\mathrm{Sol}$ motoneurons may reflect a late innervation of the pool by 5-HT fibers. Such a differential effect on maturation of the different motor pools may alter intralimb coordination and account partly for the postural and locomotor deficits observed in 5-HT-depleted animals (Myoga et al., 1995; Nakajima et al., 1998). In our study, the reduced excitability of the PBST motoneurons (innervating hip extensor/knee flexor muscles) may account for the more rostral placement of the toes in PCPA-treated animals, whereas the larger decrease in excitability in EDL-TA motoneurons compared with G-Sol motoneurons may account for the hyperextension of the distal part of the hindlimb. Thus, a major contribution of 5-HT in the fetus would be to ensure a high excitability of flexor motoneurons to preserve the flexed posture that is functionally adapted to life in utero.

In the adult, there are two main classes of motor units, fast and slow, based on the physiological properties of motoneurons and muscle fibers. Motoneurons are rather homogenous at birth (Navarrete and Vrbova, 1993), and diversification of cell types starts during the first postnatal week (Seebach and Mendell, 1996). Interestingly, the relationship between rheobase and input conductance after PCPA treatment, at P3-5, resembles that observed at P7-9 after normal development (Seebach and Mendell, 1996). Whether 5-HT depletion leads to a speeding up of the diversification of motoneuron types is unknown and requires further investigation.

\section{Serotonin and spontaneous endogenously generated activity}

Immature vertebrates exhibit spontaneous limb movements (Narayanan et al., 1971). In vitro, the spinal cord isolated from fetal or neonatal rats generates an intense spontaneous bursting activity that can be recorded from both ventral and dorsal roots (Nishimaru et al., 1996; Nakayama et al., 1999; Fellippa-Marques et al., 2000). Several factors such as a high excitability of neurons, a widespread electrotonic coupling, an overexpression of glutamate receptors (Kalb et al., 1992; Jakowec et al., 1995), and the depolarizing action of GABA and glycine (Wu et al., 1992; Gao and Ziskind-Conhaim, 1995; Nishimaru et al., 1996) contribute to trigger this activity. Spontaneous endogenously generated activities are a common feature of developing networks because they have been described in the visual system and the hippocampus (for review, see O'Donovan, 1999; Ben-Ari, 2001). Our experiments showing that the spontaneous activity was more in- tense in PCPA-treated animals than in controls suggest that developing spinal networks are sensitive to 5-HT levels.

Spontaneous endogenously generated activities are believed to play a key role in different developmental processes through the $\mathrm{Ca}^{2+}$ oscillations that they trigger in neurons (for review, see Vinay et al., 2000b). Several activity-dependent processes (Demerens et al., 1996) are sensitive to the frequency of $\mathrm{Ca}^{2+}$ oscillations, and alteration of the activity after 5-HT depletion may thereby influence neuronal development. For example, myelination has been related to the level of spontaneous activity (Stevens et al., 1998). The increased conduction velocity of motoneurons after serotonin depletion may reflect a change in total axon diameter, attributable to an increased myelination of motor axons, which begins at birth (Ziskind-Conhaim, 1988), rather than an increased diameter of the axon associated with an increased somatic growth.

In conclusion, the present results show for the first time the effects of 5-HT depletion on motoneuron development in mammals. Part of its action is likely exerted through a modulation of activity-dependent processes. Disturbances affecting 5-HT levels in the developing CNS therefore may have long-term severe consequences on the motor development of the child, especially deficits in the development of standing and walking.

\section{REFERENCES}

Ben-Ari Y (2001) Developing networks play a similar melody. Trends Neurosci 24:353-360.

Berger AJ, Bayliss DA, Viana F (1996) Development of hypoglossal motoneurons. J Appl Physiol 81:1039-1048.

Bolles RC, Woods PJ (1964) The ontogeny of behaviour in the albino rat. Anim Behav 12:427-441.

Bou-Flores C, Lajard AM, Monteau R, De Maeyer E, Seif I, Lanoir J, Hilaire G (2000) Abnormal phrenic motoneuron activity and morphology in neonatal monoamine oxidase A-deficient transgenic mice: possible role of a serotonin excess. J Neurosci 20:4646-4656.

Bregman BS (1987) Development of serotonin immunoreactivity in the rat spinal cord and its plasticity after neonatal spinal cord lesions. Brain Res 431:245-263.

Brocard F, Vinay L, Clarac F (1999a) Development of hindlimb postural control during the first postnatal week in the rat. Dev Brain Res 117:81-89.

Brocard F, Vinay L, Clarac F (1999b) Gradual development of the ventral funiculus input to lumbar motoneurons in the neonatal rat. Neuroscience 90:1543-1554.

Burnet H, Bevengut M, Chakri F, Bou-Flores C, Coulon P, Gaytan S, Pasaro R, Hilaire G (2001) Altered respiratory activity and respiratory regulations in adult monoamine oxidase A-deficient mice. J Neurosci 21:5212-5221.

Buznikov GA, Lambert HW, Lauder JM (2001) Serotonin and serotonin-like substances as regulators of early embryogenesis and morphogenesis. Cell Tissue Res 305:177-186. 
Cameron WE, Nunez-Abades PA, Kerman IA, Hodgson TM (2000) Role of potassium conductances in determining input resistance of developing brain stem motoneurons. J Neurophysiol 84:2330-2339.

Cazalets JR, Gardette M, Hilaire G (2000) Locomotor network maturation is transiently delayed in the MAOA-deficient mouse. J Neurophysiol 83:2468-2470.

Chang Q, Gonzalez M, Pinter MJ, Balice-Gordon RJ (1999) Gap junctional coupling and patterns of connexin expression among neonatal rat lumbar spinal motor neurons. J Neurosci 19:10813-10828.

Dekkers J, Becker DL, Cook JE, Navarrete R (1994) Early postnatal changes in the somatodendritic morphology of ankle flexor motoneurons in the rat. Eur J Neurosci 6:87-97.

Demerens C, Stankoff B, Logak M, Anglade P, Allinquant B, Couraud F, Zalc B, Lubetzki C (1996) Induction of myelination in the central nervous system by electrical activity. Proc Natl Acad Sci USA 93:9887-9892.

Fellippa-Marques S, Vinay L, Clarac F (2000) Spontaneous and locomotor-related GABAergic input onto primary afferents in the neonatal rat. Eur J Neurosci 12:155-164.

Fulton BP, Walton K (1986) Electrophysiological properties of neonatal rat motoneurones studied in vitro. J Physiol (Lond) 370:651-678.

Gao B-X, Ziskind-Conhaim L (1995) Development of glycine- and GABA-gated currents in rat spinal motoneurons. J Neurophysiol 74:113-121.

Gao BX, Ziskind-Conhaim L (1998) Development of ionic currents underlying changes in action potential waveforms in rat spinal motoneurons. J Neurophysiol 80:3047-3061.

Geisler HC, Westerga J, Gramsbergen A (1993) Development of posture in the rat. Acta Neurobiol Exp (Wars) 53:517-523.

Haydon PG, McCobb DP, Kater SB (1984) Serotonin selectively inhibits growth cone motility and synaptogenesis of specific identified neurons. Science 226:561-564.

Jacobs BL, Fornal CA (1993) 5-HT and motor control: a hypothesis. Trends Neurosci 16:346-352.

Jakowec MW, Fox AJ, Martin LJ, Kalb RG (1995) Quantitative and qualitative changes in AMPA receptor expression during spinal cord development. Neuroscience 67:893-907.

Jankowska E, Hammar I, Chojnicka B, Heden CH (2000) Effects of monoamines on interneurons in four spinal reflex pathways from group I and/or group II muscle afferents. Eur J Neurosci 12:701-714

Kalb RG, Lidow MS, Halsted MJ, Hockfield S (1992) $N$-methyl-Daspartate receptors are transiently expressed in the developing spinal cord ventral horn. Proc Natl Acad Sci USA 89:8502-8506.

Kerai B, Greensmith L, Vrbova G, Navarrete R (1995) Effect of transient neonatal muscle paralysis on the growth of soleus motoneurones in the rat. Dev Brain Res 85:89-95.

Kiehn O, Erdal J, Eken T, Bruhn T (1996) Selective depletion of spinal monoamines changes the rat soleus EMG from a tonic to a more phasic pattern. J Physiol (Lond) 492:173-184.

Koe BK, Weissman A (1966) p-Chlorophenylalanine: a specific depletor of brain serotonin. J Pharmacol Exp Ther 154:499-516.

Kojima T, Homma S, Sako H, Shimizu I, Okada A, Okado N (1988) Developmental changes in density and distribution of serotoninergic fibers in the chick spinal cord. J Comp Neurol 267:580-589.

Kudo N, Furukawa F, Okado N (1993) Development of descending fibers to the rat embryonic spinal cord. Neurosci Res 16:131-141.

Lakke EAJF (1997) The projections to the spinal cord of the rat during development: a time-table of descent. Adv Anat Embryol Cell Biol 135:1-143.

Lauder JM (1993) Neurotransmitters as growth regulatory signals: role of receptors and second messengers. Trends Neurosci 16:233-240.

Mazer C, Muneyyirci J, Taheny K, Raio N, Borella A, Whitaker-Azmitia $P$ (1997) Serotonin depletion during synaptogenesis leads to decreased synaptic density and learning deficits in the adult rat: a possible model of neurodevelopmental disorders with cognitive deficits. Brain Res 760:68-73.

Myoga H, Nonaka S, Matsuyama K, Mori S (1995) Postnatal development of locomotor movements in normal and parachlorophenylalanine-treated newborn rats. Neurosci Res 21:211-221.

Nakajima K, Matsuyama K, Mori F (1996) Prenatal suppression of serotonergic system results in movement disorders and hypoplesia of extensor motoneurons in newborn rats. Soc Neurosci Abstr 22:1228.

Nakajima K, Matsuyama K, Mori S (1998) Prenatal administration of para-chlorophenylalanine results in suppression of serotonergic system and disturbance of swimming movements in newborn rats. Neurosci Res 31:155-169.
Nakayama K, Nishimaru H, Iizuka M, Ozaki S, Kudo N (1999) Rostrocaudal progression in the development of periodic spontaneous activity in fetal rat spinal motor circuits in vitro. J Neurophysiol 81:2592-2595.

Narayanan CH, Fox MW, Hamburger V (1971) Prenatal development of spontaneous and evoked activity in the rat (Rattus Norvegicus Albinus) Behaviour 40:100-135.

Navarrete R, Vrbova G (1993) Activity-dependent interactions between motoneurones and muscles: their role in the development of the motor unit. Prog Neurobiol 41:93-124.

Nicolopoulos-Stournaras S, Iles JF (1983) Motor neuron columns in the lumbar spinal cord of the rat. J Comp Neurol 217:75-85.

Nishimaru H, Iizuka M, Ozaki S, Kudo N (1996) Spontaneous motoneuronal activity mediated by glycine and GABA in the spinal cord of rat fetuses in vitro. J Physiol (Lond) 497:131-143.

Nunez-Abades PA, Pattillo JM, Hodgson TM, Cameron WE (2000) Role of synaptic inputs in determining input resistance of developing brain stem motoneurons. J Neurophysiol 84:2317-2329.

O'Donovan MJ (1999) The origin of spontaneous activity in developing networks of the vertebrate nervous system. Curr Opin Neurobiol 9:94-104.

Okado N, Homma S, Ishihara R, Sako H, Kohno K (1988) Differential innervation of specific motor neuron pools by serotoninergic fibers in the chick spinal cord. Neurosci Lett 94:29-32.

Okado N, Cheng L, Tanatsugu Y, Hamada S, Hamaguchi K (1993) Synaptic loss following removal of serotoninergic fibers in newly hatched and adult chickens. J Neurobiol 24:687-698.

Pflieger J-F, Clarac F, Vinay L (2000) Effects of serotonin depletion on the maturation of ankle flexor and extensor motoneurons in neonatal rats. Soc Neurosci Abstr 26:257.5.

Rajaofetra N, Sandillon F, Geffard M, Privat A (1989) Pre- and postnatal ontogeny of serotonergic projections to the rat spinal cord. J Neurosci Res 22:305-321.

Rörig B, Sutor B (1996) Serotonin regulates gap junction coupling in the developing rat somatosensory cortex. Eur J Neurosci 8:1685-1695.

Schmidt BJ, Jordan LM (2000) The role of serotonin in reflex modulation and locomotor rhythm production in the mammalian spinal cord. Brain Res Bull 53:689-710.

Seebach BS, Mendell LM (1996) Maturation in properties of motoneurons and their segmental input in the neonatal rat. J Neurophysiol 76:3875-3885

Sillar KT, Woolston AM, Wedderburn JF (1995) Involvement of brainstem serotonergic interneurons in the development of a vertebrate spinal locomotor circuit. Proc R Soc Lond B Biol Sci 259:65-70.

Steinbusch HW (1981) Distribution of serotonin-immunoreactivity in the central nervous system of the rat-cell bodies and terminals. Neuroscience 6:557-618.

Stevens B, Tanner S, Fields RD (1998) Control of myelination by specific patterns of neural impulses. J Neurosci 18:9303-9311.

Sullivan JM, Benton JL, Beltz BS (2000) Serotonin depletion in vivo inhibits the branching of olfactory projection neurons in the lobster deutocerebrum. J Neurosci 20:7716-7721.

Tanaka H, Mori S, Kimura H (1992) Developmental changes in the serotoninergic innervation of hindlimb extensor motoneurons in neonatal rats. Dev Brain Res 65:1-12.

Tohyama I, Kameyama M, Kimura H (1988) Quantitative morphometric analysis of two types of serotonin-immunoreactive nerve fibres differentially responding to $p$-chlorophenylalanine treatment in the rat brain. Neuroscience 26:971-991.

Vinay L, Brocard F, Clarac F (2000a) Differential maturation of motoneurons innervating ankle flexor and extensor muscles in the neonatal rat. Eur J Neurosci 12:4562-4566.

Vinay L, Brocard F, Pflieger J, Simeoni-Alias J, Clarac F (2000b) Perinatal development of lumbar motoneurons and their inputs in the rat. Brain Res Bull 53:635-647.

Walton K, Navarrete R (1991) Postnatal changes in motoneurone electrotonic coupling studied in the in vitro rat lumbar spinal cord. J Physiol (Lond) 433:283-305.

Westerga J, Gramsbergen A (1992) Structural changes of the soleus and the tibialis anterior motoneuron pool during development in the rat. J Comp Neurol 319:406-416.

Wu W-L, Ziskind-Conhaim L, Sweet MA (1992) Early development of glycine- and GABA-mediated synapses in rat spinal cord. J Neurosci 12:3935-3945.

Ziskind-Conhaim L (1988) Physiological and morphological changes in developing peripheral nerves of rat embryos. Dev Brain Res 42:15-28. 